Concevoir et représenter l'utopie La diffusion du modèle des garden-cities en Grande-Bretagne,

\title{
1898-2015
}

Thinking and representing utopia. The diffusion of the model of garden-cities in Great Britain 1898-2015

\section{Stéphane Sadoux}

\section{(2) OpenEdition Journals}

Édition électronique

URL : http://journals.openedition.org/communicationorganisation/5045

DOI : 10.4000/communicationorganisation.5045

ISSN : 1775-3546

\section{Éditeur}

Presses universitaires de Bordeaux

\section{Édition imprimée}

Date de publication : 1 décembre 2015

Pagination : 25-38

ISSN : 1168-5549

Référence électronique

Stéphane Sadoux, «Concevoir et représenter l'utopie La diffusion du modèle des garden-cities en Grande-Bretagne, 1898-2015», Communication et organisation [En ligne], 48 | 2015, mis en ligne le 01 décembre 2018, consulté le 21 décembre 2020. URL : http://journals.openedition.org/ communicationorganisation/5045; DOI : https://doi.org/10.4000/communicationorganisation.5045 


\title{
Concevoir et représenter l'utopie La diffusion du modèle des garden-cities en Grande-Bretagne, 1898-2015
}

\author{
Stéphane Sadoux'
}

S'il est une difficulté à laquelle tous les gouvernements britanniques se sont heurtés depuis des décennies, c'est bien celle de la crise du logement. Les grands projets urbains lancés par Tony Blair à la fin des années 1990 n'ont pas suffi à répondre à la demande, ni à renverser la périurbanisation qui perdure depuis plus d'un siècle dans un pays où les villes sont boudées par une grande partie de la population au profit des suburbs. Confronté à ces enjeux, le gouvernement de coalition de David Cameron annonçait en 2014 la construction de deux nouvelles garden cities $^{2}$ - l'une à Ebbsfleet à l'est de Londres, l'autre à Bicester dans l'Oxfordshire. Pour le vice premier ministre Nick Clegg, il s'agissait alors de lancer un « appel aux armes aux visionnaires » afin de répondre à la crise du logement au travers de "propositions radicales et ambitieuses » (The Guardian 2014). Quelques mois plus tard, les urbanistes Nicholas Falk et David Rudlin de l'agence URBED remportaient le prestigieux Wolfson Economics Prize grâce à une réinterprétation du modèle des garden cities (Rudlin et Falk 2014).

Cet article propose d'interroger le retour de ce précédent hérité du $19^{\text {e }}$ siècle et fréquemment qualifié d'utopie. Il vise en particulier à montrer en quoi les outils de représentation déployés par Ebenezer Howard, père des garden cities, pour diffuser ce modèle en ont fait une référence incontournable du

\footnotetext{
1 Stéphane Sadoux est diplômé en urbanisme (BA(Hons), DipTP, MTP) de l'université de Newcastle, Grande-Bretagne, titulaire d'un DEA Villes et Sociétés de l'Institut d'Urbanisme de Grenoble et docteur en urbanisme et aménagement de l'université Pierre Mendes France. Il est actuellement directeur du laboratoire Cultures Constructives, Unité de recherché (LabEx) Architecture, Environnement \& Cultures Constructives et enseignant à l'Ecole Nationale Supérieure d'Architecture de Grenoble. Il a enseigné à l'Institut des Sciences de l'Environnement et à l'Institut d'Architecture de l'Université de Genève ainsi qu'à à l'Institut d'Urbanisme de Grenoble. Il a par ailleurs exercé à la Town and Country Planning Association à Londres. Ses recherches portent sur les théories et l'histoire de l'urbanisme anglo-saxon ; sadoux.s@grenoble.archi.fr

2 Le terme anglais est volontairement conservé, afin d'éviter toute confusion avec les différents types de « citésjardins » que le modèle britannique a inspiré aux quatre coins du monde.
} 
monde de l'architecture et de l'urbanisme. Il se donne également pour objectif d'interroger la dimension utopique de ce modèle.

\section{Une métaphore pour un projet}

Les garden cities sont nées de l'imagination et du travail d'Ebenezer Howard (1850-1938). Autodidacte, il quitte l'école à l'âge de quatorze ans. Après cinq années passées aux États-Unis où il exerce notamment le journalisme, il rentre dans son Angleterre natale en 1876 et est employé à Londres comme sténographe au Parlement où il participe à la transcription des débats. Immergé dans les grandes questions de société qui agitent alors le pays, il porte un intérêt particulier à l'amélioration des conditions de vie des populations. Dans leur " dictionnaire des alternatives ", Parker, Fournier et Reedy (2007) nous rappellent que chaque époque génère ses propres utopies qui reflètent ses injustices et inquiétudes. Dans l'Angleterre de la fin du $19^{\mathrm{e}}$ siècle, Howard constate que ni la ville industrielle ni la campagne, victime d'un exode rural massif, n'offrent un cadre de vie acceptable. L'analyse qu'il fait est restituée dans l'ouvrage qu'il publie en 1898 : To-Morrow : A Peaceful Path to Real Reform. L'une des grandes forces de ce livre réside dans la dimension didactique de ses différentes illustrations. L'une d'entre elles, intitulée " les trois aimants ${ }^{3}$ ", est la pièce graphique la plus diffusée dans le domaine de l'urbanisme.

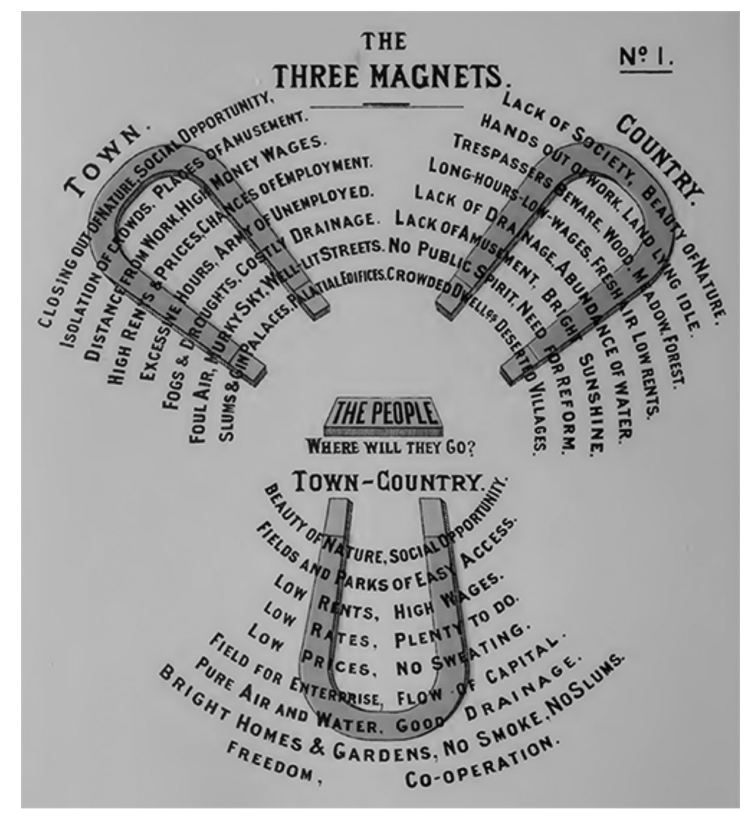

Figure 1 : The three magnets. Source : Howard (1898)

3 The three magnets. 
La métaphore de l'aimant est employée pour illustrer ce qui « attire " les populations. Le choix de Howard démontre toute la force de la métaphore comme outil de révélation et de compréhension de l'environnement bâti relevé par Fez-Barringten (2010) qui nous rappelle par ailleurs que la métaphore, issue de la linguistique, est largement employée dans les disciplines liées à l'espace. Elle permet en effet de comprendre un ensemble d'enjeux complexes et déstructurés à travers un « sujet » qui, à l'inverse, est structuré. La familiarité du sujet convoqué (ici, l'aimant), permet à Howard de représenter et de communiquer sa pensée de manière claire et concise grâce à une image pouvant être comprise par tout un chacun. Placée en première page de l'ouvrage, elle offre une représentation synthétique des forces, faiblesses, opportunités et menaces qui caractérisent la ville et la campagne, mais également les atouts du projet alternatif qu'il propose au travers d'un nouveau type d'établissement humain : les garden cities. Si les villes proposent notamment des emplois, des salaires plus attractifs et des loisirs, elles sont fortement polluées et ne permettent aucun rapport à la nature. Les logements qu'on y trouve sont de mauvaise qualité et loués au prix fort. Les campagnes offrent l'air pur, des paysages de qualité et des loyers abordables mais peu d'emplois, de vie sociale et aucun système d'assainissement. C'est sur la base de cette confrontation entre ville et compagne que Howard propose les garden cities qui, d'après lui, réunissent le meilleur de ces deux types d'environnements sans leurs inconvénients respectifs. Les populations y trouveraient en particulier ainsi un cadre verdoyant, des logements de qualité à bas prix, des emplois et des salaires élevés. Germán Solinis (2006) nous rappelle que l'urbanisme tend à opposer deux images de la ville : l'une, négative, qui met en avant ses défauts et un certain désordre ; l'autre positive, assortie de propositions pour remettre de l'ordre. La représentation des trois aimants illustre cette tendance, mais démontre également que le modèle des garden cities est avant tout un projet de société économique, social, et environnemental qu'il convient d'énoncer avant de le spatialiser. Ce projet vise à répondre à des enjeux complexes. Söderström (1996) nous rappelle que les représentations de la ville traitent et communiquent la réalité par le biais d'un ensemble de procédés, notamment la sélection, la schématisation et la synthèse : les pièces graphiques produites par les architectes et les urbanistes sont donc des cristallisations de leurs pensées. Les trois aimants de Howard sont, pour reprendre l'expression de Söderström, empreints d'une "efficacité interne »: ils permettent de passer d'une réalité complexe à sa figuration simplifiée. La communication visuelle, largement utilisée dans l'architecture et l'urbanisme, permet en effet de décrire et de comprendre la complexité (Laseau, 2001). C'est sans doute l'efficacité interne du diagramme de Howard qui a contribué à sa très large diffusion, au-delà du cercle des professionnels du territoire. 


\section{Une utopie pour comprendre et agir}

Les travaux d'Anne Coste $(1997,2008)$ portant sur les théories et l'histoire de l'architecture nous rappellent qu'un modèle peut servir à comprendre, à représenter ou à concevoir. La construction théorique du modèle de Howard repose en premier lieu sur une analyse fine du contexte de l'époque qu'il s'agit de cerner. La première fonction que Coste attribue au modèle (permettre de comprendre) n'est pas sans rappeler le point de vue développé par Robert Tally (2013) au sujet de l'utopie qui, d'après lui, ne doit pas être considérée comme une société ou une condition idéale mais plutôt comme une méthode grâce à laquelle l'on pourrait appréhender une situation. Il s'agirait donc d'un moyen mis au service d'une " modélisation du monde ${ }^{4}$ ", reflet d'une volonté de se situer dans l'espace et dans l'histoire. L'utopie permettrait en d'autres termes de comprendre une situation et de se doter des outils pour la faire évoluer : il est en effet essentiel de voir le monde tel qu'il est si l'on veut envisager de le changer. Dans la même veine, Amir Ganjavie (2015) suggère que les urbanistes utopistes proposent des modèles provocateurs qui deviennent une forme de résistance visant à questionner le monde et à proposer des alternatives. Notons enfin le point de vue de Howard Segal (2012) pour qui l'objectif d'une utopie n'est pas de prévoir l'avenir, mais plutôt d'améliorer le présent et le monde réel. Si nous considérons qu'une utopie ne peut être conçue que sur la base d'un décryptage et d'une bonne compréhension de la réalité qu'il a vocation à bouleverser, il est peu étonnant de voir le modèle de Howard régulièrement qualifié d'utopie ${ }^{5}$. Pour Robert Fishman (1982) notamment, l'héritage du père des garden cities relève effectivement de l'utopie, mais pas au sens péjoratif que l'on pourrait attribuer à un rêve irréalisable : il s'agirait au contraire de le comprendre tel que Karl Mannheim l'entendait, à savoir un programme d'action cohérent qui dépasse une situation immédiate afin de rompre avec l'ordre établi (Mannheim, 1936). De la même manière, Ganjavie considère l'utopie de Howard comme un " programme d'action cohérent résultant d'une réflexion profonde visant à transcender la situation immédiate » (Ganjavie 2015 : 92).

\section{Un modèle d'organisation spatiale alternative}

Ebenezer Howard n'est pas architecte, pas plus qu'il n'est urbaniste mais son ouvrage, publié une nouvelle fois en 1902 sous le titre Garden Cities of To-Morrow, démontre tout l'intérêt qu'il portait à l'organisation spatiale des villes et des régions. Rappelons qu'entre le début du $18^{\mathrm{e}}$ siècle et les années 1840, la population de Londres passe de 630000 habitants à plus de deux millions. Dans la capitale du pays comme dans ses autres métropoles

4 Tally emploie la phrase « mapping the world». Nous proposons ici de traduire le terme mapping par modéliser car il ne s'agit pas tant de cartographier. Tally renvoie d'ailleurs à la notion de « cognitive mapping » de Jameson. 5 Le projet de Howard figure par exemple dans l'ouvrage de Dennis Hardy (2000) intitulé Utopian England, ainsi que dans Urban Utopias de Malcom Miles (2008) 
industrielles, la logique de croissance est alors monocentrique. Par opposition, le modèle de Howard est hiérarchisé et repose sur un principe que nous qualifierions aujourd'hui de polycentrique : l'objectif est de renverser la tendance à la dominance d'une métropole sur une région. Howard envisage que la première garden city, désignée par le nom " central city ", accueillerait 58000 habitants. Une fois cette limite atteinte, de nouvelles garden cities, dont la population serait de 32000 habitants, seraient érigées autour d'elles telles des satellites. Le modèle d'urbanisation pensé par Howard est un système : les cités seraient reliées entre elles, non seulement par des axes routiers mais également et par-dessus tout par un réseau ferré, l'intermunicipal railway. Ce principe de croissance est une fois encore explicité par un diagramme (Figure 2) représentant les principes d'urbanisation à l'échelle de la région. L'organisation spatiale proposée pour l'échelle de la garden city individuelle est pour sa part mise en évidence dans un autre schéma (Figure 3) lequel fournit des indications plus nombreuses, notamment en termes d'occupation des sols. Mais une fois encore, Howard souhaite exprimer des principes dans leurs grandes lignes. En témoigne le nota bene inséré en bas à droite, dont la typographie laisserait presque croire qu'il s'agit d'un titre. Et pour cause, Howard tenait à mettre le lecteur en garde : cette représentation ne vise en aucun cas à proposer un modèle qu'il s'agitait de répliquer fidèlement en n'importe quel lieu. Bien au contraire, l'annotation rappelle que le plan de la cité ne peut être dessiné avant que le site d'implantation ne soit sélectionné. Le modèle a donc vocation à s'inscrire dans un territoire auquel il doit s'adapter.

À travers ces deux pièces graphiques transparait une autre fonction du modèle de Howard : il s'agit ici, pour reprendre les termes d'Anne Coste, d'un modèle pour représenter mais également pour concevoir. Le rôle de ces diagrammes, en tant que base de conception, démontre la volonté de Howard de voir son utopie être concrétisée.

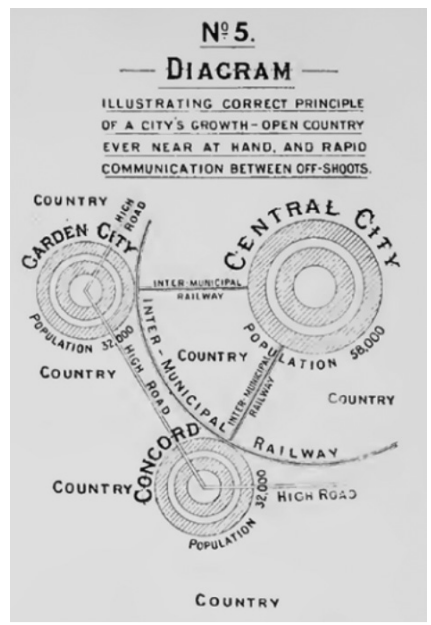

Figure 2 : Principes de croissance d'une ville. Source : Howard (1898) 


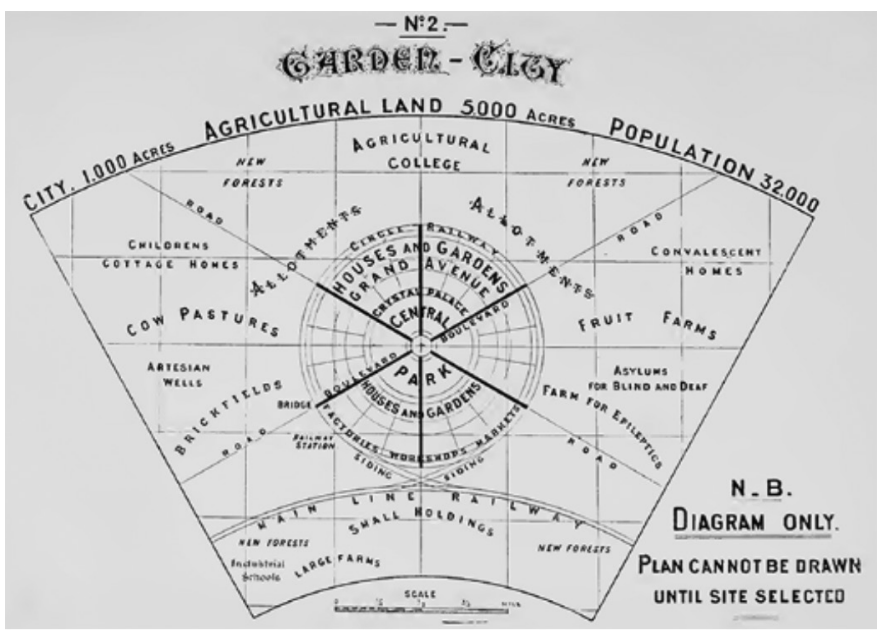

Figure 3 : Schéma d'aménagement d'une garden city

Source : Howard (1898)

\section{Un modèle de gestion du foncier et de gouvernance}

Les mondes meilleurs incarnés par les utopies urbaines ne sont pas seulement des environnements construits : ils sont également et par-dessus tout des systèmes sociaux. L'historien de la ville Gordon Cherry soulignait ainsi que les avancées de l'urbanisme du vingtième siècle ont reposé sur deux principaux antécédents : l'un relève de la morphologie urbaine et de la ville idéale en termes architecturaux et urbanistiques, l'autre concerne la quête de la communauté idéale (Cherry 1970). Le modèle des garden cities s'attache à ces deux dimensions et accorde une attention toute particulière à la question du foncier dans ses dimensions économiques et sociales mais également à la gestion de la cité. Dans le projet de Howard, les terres nécessaires à la construction des garden cities seraient achetées grâce à des emprunts. Il s'agirait de terres agricoles acquises au prix du marché. La construction puis la croissance progressive de la cité contribueraient à terme à la hausse de la valeur du foncier et donc des loyers payés par les résidents ${ }^{6}$. C'est précisément cette appréciation qui permettrait non seulement de rembourser les emprunts et de payer des intérêts aux créanciers, mais également de dégager des bénéfices lesquels seraient réinvestis au profit de la communauté de la garden city. Hall et Ward (2014) rappellent qu'une fois les emprunts et les intérêts remboursés, c'est un véritable welfare state local qui serait instauré : les coûts des services aux personnes (notamment âgées et malades), de l'entretien de la garden city et des équipements seraient couverts par les loyers, dispensant les résidents de toute forme d'imposition. Hall et Ward soulignent également que la

6 À l'époque, et à titre d'exemple, Hall et Ward (2014) rappellent qu'un loyer de $£ 4$ à l'acre aurait été considéré comme élevé alors qu’à Londres, le prix à payer pour la même surface aurait été de $£ 30000$. 
part très importante de son ouvrage que Howard accorde à la dimension financière des garden cities avait notamment pour objectif de convaincre les investisseurs potentiels : son utopie urbaine était également un redoutable projet de marketing. Ici encore transparait clairement la volonté de Howard de concrétiser et de pérenniser sa vision.

La gouvernance de la garden city devait pour sa part être fondée sur deux principes : la liberté et la coopération - deux mots que l'on trouve inscrits en bas du diagramme des trois aimants. Elle serait incarnée par deux structures constituant le «Board of Management» : en premier lieu le Central Council, propriétaire de la garden city qui percevrait les loyers, en second lieu le Department notamment chargé de contrôler les finances et d'assurer des missions relevant par exemple de l'éducation ou de l'ingénierie. S'ajouterait à ces structures la Limited Dividend Company, une société immobilière aux dividendes limités, dont la mission concernerait le retour sur investissement. Howard n'avait semble-t-il toutefois pas prévu que des conflits entre le Board of Management et la Limited Dividend Company étaient probables, voire inévitables.

\section{Du modèle théorique à la garden city construite : la concrétisation de l'utopie}

Pour publier son ouvrage à compte d'auteur en 1898, Howard dû emprunter $£ 50$. L'année suivante, fort du succès de ce livre, il fondait la Garden Cities Association pour assurer la promotion de son modèle. En 1903, ses soutiens lui avançaient plus de $£ 100000$ pour lancer la construction de Letchworth, la première Garden City. À l'instar d'autres grands noms de l'urbanisme, Howard nous a légué un projet qui prend plusieurs formes : écrite, à travers son ouvrage ; dessinée par le biais des multiples diagrammes qui illustrent ce livre mais également construite, puisque ce sont au final deux garden cities qui sont érigées à Letchworth puis Welwyn au début du vingtième siècle.

C'est aux architectes Raymond Unwin et Barry Parker que Howard fait appel pour concrétiser l'utopie à travers la conception et la réalisation de Letchworth. Depuis quelques années, les deux hommes expérimentaient de nouvelles formes architecturales et urbaines à New Earswick, sorte de garden city érigée pour les industriels et chocolatiers Rowntree. Le plan de la ville évoque déjà les garden cities à venir : les cottages, qui répondent à des normes précises, notamment en matière d'ensoleillement, sont disposés en rangées, ou groupés autour d'espaces verts communaux ou le long de voies de circulation piétonnes. À Letchworth comme à Welwyn, la forme urbaine privilégiée est le close dont l'espace intérieur «s'oppose à l'espace spécifiquement public de la rue par une fermeture particulière : rétrécissement, haie ou même porte. Et l'opposition public/privé fonctionne de nouveau à l'intérieur du close, mais avec les réductions qu'impose une appropriation collective de cet intérieur » (Panerai, Castex et Depaule 1997, p. 71). 


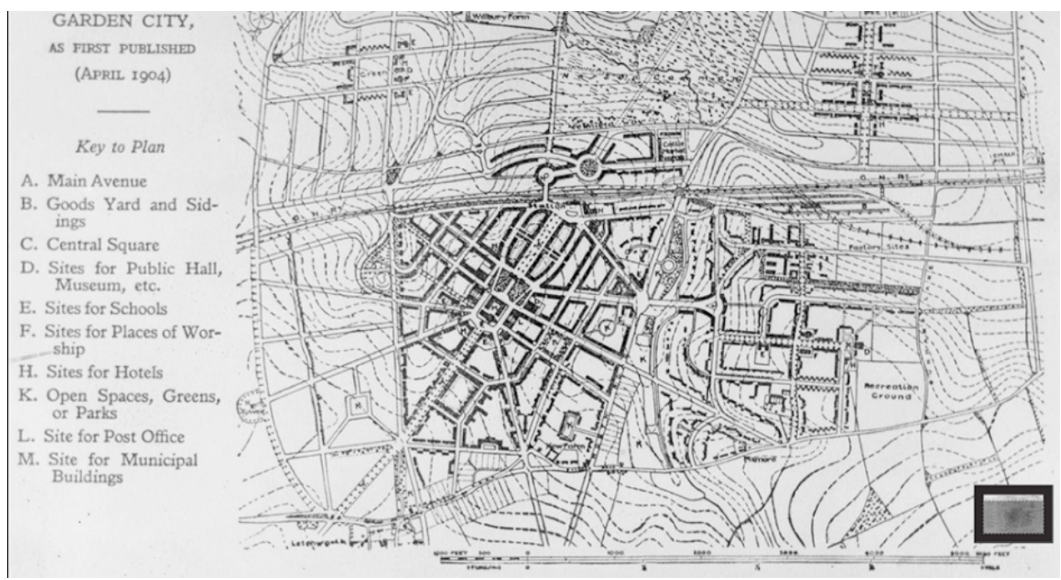

Figure 4 : Plan de Letchworth dessiné par Raymon Unwin et Barry Parker en 1904. Reproduit avec l'autorisation de The Letchworth Heritage Foundation

Quelques années plus tard, en 1909, alors même que la Grande-Bretagne voit promulguée sa première loi en matière d'urbanisme ${ }^{7}$ et fondée la première unité de formation dans ce domaine ${ }^{8}$, Raymond Unwin publie son ouvrage intitule Town Planning in Practice : an introduction to the art of designing cities and suburbs, un véritable manuel de conception architecturale et urbaine reprenant tous les canons de la ville traditionnelle et pittoresque mis en œuvre à Letchworth. Dans cet ouvrage, Unwin propose des modèles pour concevoir, en particulier des variations sur le thème du close. Les nombreuses illustrations du livre, exprimant les relations entre édifices, voiries, parcellaires, espaces publics et espaces privés, en ont un catalogue à destination des architectes et urbanistes. Une fois encore il s'agit de se donner les moyens de mettre en œuvre et donc de concrétiser l'utopie imaginée par Howard.

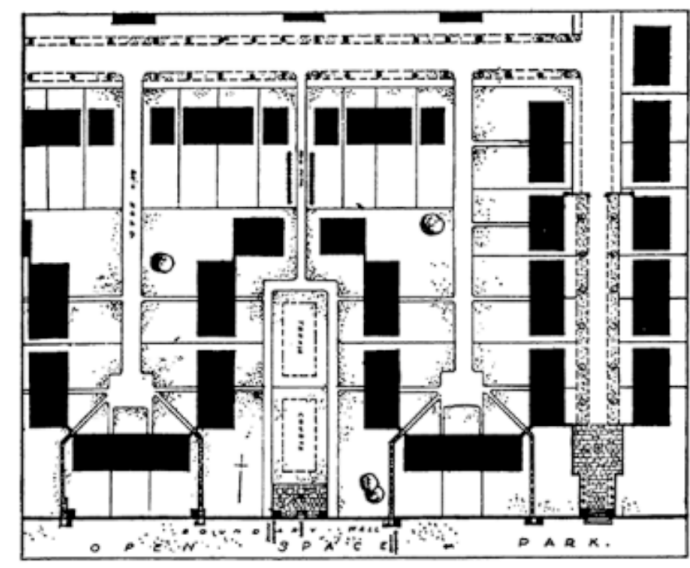

7 Le Housing, Town Planning etc. Act (1909).

8 Le Department of Civic Design de l'Université de Liverpool.
Figure 5 : Exemple d'aménagement extraitde l'ouvrage de Raymond Unwin. Source : Unwin (1909) 


\section{Pérenniser et réinterpréter l'utopie : le modèle revisité au $21^{\mathrm{e}}$ siècle}

Alexander Cuthbert (2007) a attiré notre attention sur la quasi-absence de nouvelles théories marquantes dans le domaine de l'urbanisme depuis une cinquantaine d'années. Dans la même veine, David Harvey (2000) relevait le peu de valeur que la société postmoderne attribue à l'utopie, avec pour conséquence une difficulté à renouveler ses points de vue sur la ville contemporaine. Déjà en 1999, dans son ouvrage intitulé The End of Utopia, Jacoby déclarait la mort de la pensée utopique et l'attribuait à l'essoufflement des politiques à offrir des alternatives. Pourtant, c'est bien un modèle utopique que le gouvernement britannique convoquait récemment en le brandissant comme une réponse possible à la crise du logement. S'il en est ainsi, c'est sans doute parce que le projet de Howard suit les préceptes d'un urbanisme de qualité tel que nous l'entendons aujourd'hui : la garden city a notamment une dimension qui permet à ses résidents de se déplacer à pied mais elle est également remplie par et entourée d'espaces non bâtis et donc de biodiversité (Hall et Ward 2014). Dans l'état actuel des choses, toutefois, il semble que les garden cities sont avant tout mobilisées par le gouvernement comme un modèle d'audace: les modalités de sa mise en œuvre restent encore très floues, tout comme la légitimité de l'appellation "garden cities»(Sadoux 2015). Les déclarations du gouvernement laissent en effet penser que la question foncière, au cœur de la pensée utopique de Howard, n'est pas véritablement traitée. Bien sûr, il est aujourd'hui inconcevable qu'une garden city soit érigée selon les mêmes modalités que celles imaginées par Howard, mais la question qu'il posait en son temps reste fondamentalement la même : comment s'assurer qu'une grande partie de la plus-value générée par la construction et l'aménagement soit réinvesti au bénéfice de la communauté plutôt que des investisseurs ou promoteurs?

Le gouvernement de David Cameron n'a toutefois pas le monopole de la réinterprétation contemporaine du modèle de Howard. Outre l'action permanente de l'association ${ }^{9}$ fondée par Howard, d'autres se sont saisis du modèle : le prestigieux Wolfson Economics Prizeinvitait en 2014 à le réinterpréter. Les urbanistes Nicholas Falk et David Rudlin, lauréats du concours, ont proposé une réponse dont les grandes lignes méritent d'être relevées tant elles renvoient aux représentations produites par Howard en son temps. L'analyse et la synthèse des enjeux économiques, sociaux et environnementaux actuels sont, dans les travaux de Falk et Rudlin, représentés sous la forme d'une version contemporaine des trois aimants. La comparaison de l'illustration reproduite dans l'ouvrage de Howard et celle proposée par Falk et Rudlin plus d'un siècle après démontre la pertinence du mode de représentation imaginée par le père des garden cities, fondé sur la mise en tension deux types d'environnements jugés inadaptés et la proposition conjointe d'une alternative. La ville et la

$9 \mathrm{La}$ Garden Cities Association est aujourd'hui connue sous le nom de Town and Country Planning Association. 
campagne qui figurent sur la planche de Howard ont laissé la place à l'inner city et aux suburbs, et la garden city a été remplacée par l'urban neighbourhood.

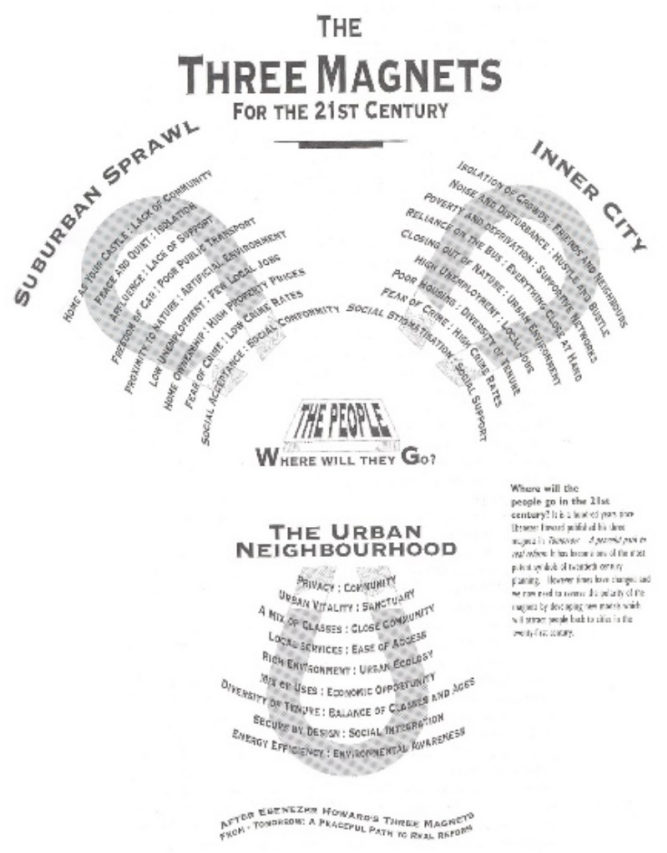

Figure 6 : The Three Magnets revisités par David Rudlin et Nicholas Falk.

Reproduit avec l'autorisation de David Rudlin et Nicholas Falk, agence URBED

Lorganisation spatiale du modèle de Falk et Rudlin s'appuie elle aussi sur le schéma de Howard, mais il démontre la capacité du modèle hérité du $19^{\text {e }}$ siècle à s'adapter à des villes existantes plutôt que d'être exclusivement mis au service de la construction de cités nouvelles. Le snowflake plan ${ }^{10}$ vise ainsi à démontrer la manière dont la construction polycentrique du territoire proposée par Howard en son temps pourrait permettre à des villes existantes de doubler leur population par un processus conjoint de densification et d'extensions urbaines associé à la construction de nouveaux villages et villessatellites. Quant à la question foncière, Falk et Rudlin offrent des pistes en imaginant que les terrains nécessaires à la construction de nouvelles garden cities puissent faire l'objet d'un rachat au prix du marché auquel s'ajouterait un dédommagement raisonnable pour leurs propriétaires. 


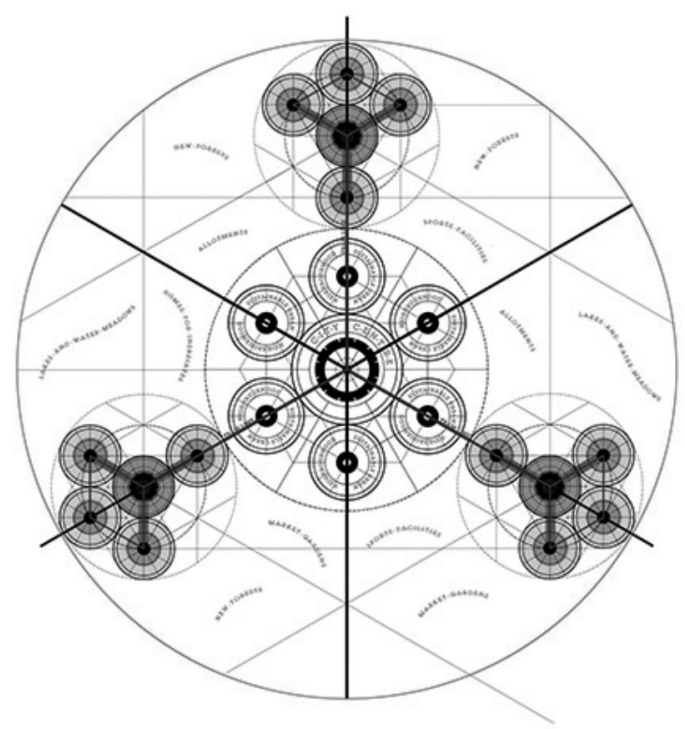

Figure 7 : Snowflake Plan

Reproduit avec l'autorisation de David Rudlin et Nicholas Falk, agence URBED

Les théories d'Ernst Bloch nous enseignent que la concrétisation de l'utopie passe notamment par une espérance instruite (docta spes), en d'autres termes par une connaissance des précédents et de leurs potentialités. Le projet de Howard, que lui-même définissait comme une " combinaison unique de propositions ", était effectivement fondé sur l'articulation de théories existantes assemblées en un tout cohérent et inédit. Ganjavie (2015) nous rappelle que Bloch encourageait à être attentif aux expériences du passé, à essayer d'y trouver des impulsions utopiques ou « surplus culturels » qui contiendraient des désirs insatisfaits : le fait qu'ils n'aient pas été testés implique qu'ils pourraient être pertinents pour la société future. La pensée de Bloch repose en effet sur l'appréhension simultanée de trois temporalités : l'analyse du passé, qui contient à la fois des erreurs à ne pas reproduire et des potentiels irréalisés, apporte des éclairages sur le présent et ouvre la voie à un avenir meilleur. Le passé est ici perçu comme un « répertoire de possibles qui sont autant d'options au service d'actions futures "; ce qui aurait pu être possible l'est donc toujours et c'est à travers la conscience anticipatoire qu'est initiée l'action (Kellner 1997 : 81). À travers leur projet, Falk et Rudlin adoptent une telle posture, et proposent de tester à plus grande échelle l'utopie de Howard dont les seules véritables concrétisations restent à ce jour les garden cities de Letchworth et Welwyn. Pour ces deux urbanistes, l'objectif est également de confronter le modèle de Howard aux villes existantes, chose que Howard n'avait pas envisagée. 


\section{Conclusion}

Dans son ouvrage intitule The Story of Utopia, l'historien américain Lewis Mumford (1923) affirme d'une part que l'utopie est depuis bien longtemps synonyme d'irréel et d'impossible, d'autre part que ce sont nos utopies qui « rendent le monde supportable » (Mumford, 1923, p. 15). Il existe d'après lui deux types d'utopies. Les premières, qui relèvent de l'évasion, laissent le monde tel qu'il est : elles ne sont que des «îles enchantées » et ne permettent donc pas de s'attaquer à la réalité telle qu'elle est ; les secondes, qualifiées d'utopies de reconstruction, visent au contraire à changer le monde (Mumford 1923 : 20). Le projet de Howard incarne le parfait exemple de cette seconde catégorie. Frederic Jameson a pour sa part souligné que nous imaginons habituellement l'utopie comme un lieu, ou plutôt " comme un non-lieu qui ressemble à un lieu » (Jameson $2010: 21)$. Il rappelle que des nouvelles formes urbaines peuvent pour les urbanistes constituer de nouveaux modèles : elles deviennent d'une certaine manière des méthodes. Ces propos sont parfaitement illustrés par le modèle de Howard et sa réinterprétation récente par Falk et Rudlin : le siècle écoulé entre la production du modèle original et son adaptation contemporaine a démontré que le projet de Howard et ses représentations offrent en définitive une méthode de travail pour les urbanistes du $21^{1 \text { ème }}$ siècle et des outils pour imaginer un futur meilleur.

Dans un article récent, Charles Hoch (2014) examinait les moyens mobilisés pour la mise en œuvre de pensées urbanistiques. Il soulignait que l'utopie, le scénario et le plan affichent des caractéristiques similaires dans la mesure où elles offrent toutes « des conseils imaginatifs " proposant une " évaluation de l'avenir comme prélude à l'action ». Le projet utopique de Howard, rédigé sous forme d'un ouvrage et spatialisé sous forme de diagrammes, les plans de Unwin ou encore le scénario de Rudlin et Falk illustrent la pérennité et la force des outils de représentation qui contribuent à la diffusion et à la communication du projet howardien. Mais très peu de modèles ont ainsi traversé les âges. Certains diront que le monde a changé et que la pensée de Howard nest plus d'actualité. Mais si les garden cites sont aujourd'hui convoquées par des professionnels reconnus et un gouvernement en quête d'alternatives, c'est sans doute parce que les enjeux identifiés par Howard en son temps (notamment le rapport à la nature, le foncier, la mobilité) sont aujourd'hui encore au cœur de toutes les préoccupations. Les garden cities du $21^{\mathrm{e}}$ siècle, qui ne sont aujourd'hui que des projets, ont au moins le mérite d'être adossées à un corpus méthodologique et à des précédents qui en font des utopies contemporaines « concrétisables». 


\section{BIBLIOGRAPHIE}

CHERRY Gordon E. (1970), Town Planning in its Social Context, Londres, Leonard Hill.

COSTE Anne (1997), « Le modèle en architecture : entre rétrospective et prospective », Cabiers de la Recherche Architecturale, n 40 «Imaginaire technique », p. 19-28.

COSTE Anne (2008), "Quel sens en architecture pour le polysémique terme de modèle ? ", Modèle, références et analogie dans la conduite à projet, Actes du séminaire doctoral inter-écoles d'architecture Rhône-Alpes, Espace, matière et société, 2008, p. 7286.

CUTHBERT Alexander R. (2007), « Requiem for an era. Review and critique of the last 50 years », Urban Design International, Vol. 12, n 4, p. 177-223.

FEZ-BARRINGTEN Barie (2012) Architecture:The Making of Metaphors, Newcastleupon-Tyne, Cambridge Scholars Publishing.

FISHMAN Robert (1982), Urban Utopias in the Twentieth Century. Ebenezer Howard, Frank Lloyd Wright, Le Corbusier, Cambridge, Mass., The MIT Press.

GANJAVIE Amir (2015), "On the future of urban design : Fabricating the future through Bloch's utopians ", Planning Theory, Vol. 14, n 1, p. 90-108.

JAMESON Frederic (2010) Utopia as Method, or the Uses of the Future, in GORDIN Michael, TILLEY Helen, PRAKASH Gyan (dir.) Utopia / Dystopia. Conditions of Historical Possibility, Princeton, Princeton University Press, p. 21-44.

HARDY Dennis (2000), Utopian England : Community Experiments 1900-1945, Londres, E\&F Spon.

HARVEY David (2000), Spaces of Hope, Berkeley, University of California Press.

HOWARD Ebenezer (1898), To-Morrow : A Peaceful Path to Real Reform, Londres, Swan Sonnenschein.

JACOBY Russell (1999), The End of Utopia, New York, Basic Books.

HALL Peter et WARD Colin (2014), Sociable Cities : the 21st Century Reinvention of the Garden City, Londres, Routledge.

HOCH Charles (2014), «Utopia,scenario and plan: A pragmaticintegration »,Planning Theory, publié en version électronique le 20 janvier 2014, doi:10.1177/1473095213518641.

KELLNER Douglas (1997), "Ernst Bloch, Utopia and Ideology Critique ", in DANIEL Jamie Owen, MOYLAN Tom (dir.) Not Yet. Reconsidering Ernst Bloch, New York, Verso, p. 80-96.

LASEAU Paul (2001) Graphic Thinking for Architects E Designers, 3rd edition, New York, John Wiley \& Sons.

MANNHEIM Karl (1936), Ideology and utopia, Londres, Routledge.

MILES Malcom (2008), Urban Utopias : The built and sociale architectures of alternative settlements, Londres, Routledge.

MUMFORD Lewis (1923), The Story of Utopias. Ideal Commonwealths and Social Myths, Londres, George G. Harrap \& Co Ltd PANERAI Philippe, CASTEX Jean, DEPAULE Jean-Charles (1997), Formes urbaines: de l'îlot à la barre, Marseille, Éditions Parenthèses. 
PARKER Martin, FOURNIER, Valérie et REEDY Patrick (2007), The Dictionary of Alternatives : Utopianism and Organization, Londres, Zed Books.

RUDLIN David et FALK Nicholas (2014), Uxcester garden city. Submission to the 2014 Wolfson Economics Prize, Manchester, URBED.

SADOUX Stéphane (2015, à paraître), « Ni ville, ni suburbs : le retour des garden cities en Grande-Bretagne », Revue Socio-anthropologie.

SEGAL Howard P. (2012), Utopias : A Brief History From Ancient Writings to Virtual Communities, Chichester, Wiley Blackwell.

SODERSTROM Ola (1996) « Paper Cities : Visual Thinking in Urban Planning », Ecumene, Vol. 3, n 3, p. 249-281.

SOLINIS Germán (2006), "Utopia, the Origins and Invention of Western Urban Design », Diogenes, Vol. 53, n 1, p. 79-87.

Résumé : Pour répondre à la crise du logement, le gouvernement britannique annonçait en 2014 la construction de deux nouvelles garden cities. La même année, les urbanistes David Rudlin et Nicholas Falk remportaient le prestigieux Wolfson Economics Prize grâce à une réinterprétation contemporaine de ce modèle imaginé par Ebenezer Howard en 1898. Cet article propose d'interroger le retour de ce précédent hérité du $19^{\mathrm{e}}$ siècle et fréquemment qualifié d'utopie. Il vise en particulier à montrer en quoi les outils de représentation déployés par Howard pour diffuser et assurer la promotion de ce modèle en ont fait une référence incontournable du monde de l'architecture et de l'urbanisme. Il se donne également pour objectif d'interroger la dimension utopique de ce modèle à la lumière d'un cadre théorique pluridisciplinaire.

Mots-clés : Garden city, Ebenezer Howard, urbanisme, utopie.

Abstract : In 2014, the British government announced that two new garden cities will be built to address the housing crisis. The same year, town planners David Rudlin and Nicholas Falk won the prestigious Wolfson Economics Prize for their reinterpretation of the model developed by Ebenezer Howard in 1898. This paper aims at reflecting upon the return of this 19 th century precedent which is often referred to as a utopia. In particular, it seeks to show how the representation methods used by Howard to disseminate and promote this model contributed to making it a key reference in the fields of architecture and town planning. This paper also aims at discussing the utopian dimension of this model based on a cross-disciplinary theoretical framework.

Keywords : Garden city, Ebenezer Howard, town planning, utopia. 